\title{
Course Recommendations in Online Education Based on Collaborative Filtering Recommendation Algorithm
}

\author{
Jing $\mathrm{Li}^{1}$ and Zhou Ye $\mathbb{1}^{2}$ \\ ${ }^{1}$ Laboratory Center of Economics and Management, Zhejiang University of Finance and Economics, Hangzhou, \\ Zhejiang 310018, China \\ ${ }^{2}$ Office of Academic Affairs, Zhejiang University of Finance and Economics, Hangzhou, Zhejiang 310018, China
}

Correspondence should be addressed to Zhou Ye; yezhou@zufe.edu.cn

Received 30 October 2020; Revised 10 December 2020; Accepted 16 December 2020; Published 28 December 2020

Academic Editor: Wei Wang

Copyright (c) 2020 Jing Li and Zhou Ye. This is an open access article distributed under the Creative Commons Attribution License, which permits unrestricted use, distribution, and reproduction in any medium, provided the original work is properly cited.

\begin{abstract}
In this paper, a personalized online education platform based on a collaborative filtering algorithm is designed by applying the recommendation algorithm in the recommendation system to the online education platform using a cross-platform compatible HTML5 and high-performance framework hybrid programming approach. The server-side development adopts a mature B/S architecture and the popular development model, while the mobile terminal uses HTML5 and framework to implement the function of recommending personalized courses for users using collaborative filtering and recommendation algorithms. By improving the traditional recommendation algorithm based on collaborative filtering, the course recommendation results are more in line with users' interests, which greatly improves the accuracy and efficiency of the recommendation. On this basis, online teaching on this platform is divided into two modes: one mode is the original teacher uploads recorded teaching videos and students can learn by purchasing online or offline download; the other mode is interactive online live teaching. Each course is a separate online classroom; the teacher will publish online class information in advance, and students can purchase to get classroom number and password information online.
\end{abstract}

\section{Introduction}

With the exponential growth of online educational resources, information is changing from text form to multimedia forms such as pictures, audio, video, and online live broadcast; the quality of information and how to screen information have become an urgent problem to be solved [1]. Particularly, in a fast-paced life with great pressure of work and study, the accuracy and speed of information acquisition are particularly important. In such a social environment, providing users with accurate and efficient information has become the main task for all walks of life [2]. Websites have made some simple responses to this situation, but, in most cases, users do not know exactly what they want. Many of these problems have led to the development of new technologies and the creation of personalized recommendation technologies to address the accuracy and efficiency of information acquisition [3]. The core idea is to recommend the information that the user needs to meet the characteristics of different users [4]. To strengthen the user experience, many websites nowadays take personalized recommendations as to the main push. As an effective means of solving information overload, personalized recommendations are also of great importance to online education platforms [5]. For example, when the number of user scores in a MOOC is small, using a user-based collaborative filtering recommendation algorithm can help explore the user's potential preferences and make more accurate recommendations [6]. In this era of information explosion, both the creator of information and the consumer of information face severe challenges, with the creator's main challenge being how to make the information he or she produces shine and the consumer's difficulty in quickly locating the information he or she needs amidst the vast amount of information [7]. In this case, the first to appear was classified navigation, which is widely used in many 
scenarios, leading users to quickly find the information they need, but also clarifies the directory structure of the site or platform and the relationship between the various levels, equivalent to a map, so that users do not get lost in the information and read the information [8]. Then, came the emergence of search engines, which is a retrieval technology that collects and processes information according to users' needs and corresponding algorithms and applies specific strategies to retrieve some specific information from the Internet and feed it back to users, to improve the speed at which people can access and collect information [9].

With the rise of artificial intelligence in recent years, the government and all occupations have vigorously developed artificial intelligence to promote the development of society and technological progress [10]. Relying on the advantage that the Internet can transcend time and space, the traditional teaching model has gradually changed, the way of learning knowledge is also richer and multilevel, and education has become simpler and more effective. The network of online education began to flourish and become popular [11]. The development of online education and the level of technological development are closely related to the change of educational philosophy, the upgrade of people's demand for user education, and the change of lifestyle [12]. Grouplets' research group proposed the key technology of the recommendation system: collaborative filtering, which is the key technology of online education [13]. At the same time, group Lens was introduced as an automated recommendation system [14]. The concept of personalized recommendations was first introduced at the American Association for Artificial Intelligence with the personalized navigation system Web Watcher by Robert Armstrong and others from Carnegie Mellon University and LIRA by Marko and others from Stanford University [15]. Since then, personalized recommendations have begun to flourish. A good recommendation system gives users a sense of belonging, makes them trust the system, and provides them with good personalized service [16]. Recommendation systems have been tested and perfected over the years, and today they are found in many fields, social media platforms, e-commerce platforms, video platforms, news media, and Internet advertising. Kizilcec et al. have deconstructed learner course engagement through MOOC learner data and proposed a scalable and information-rich taxonomy that identifies engagement trajectories in MOOCs for use in predicting what students will learn next for guided learning [17]. Macina et al. looked at question recommendations, examining respondents' background knowledge and question fit, as well as their willingness to answer questions, to develop a new model of student activity that prompts more participation [18]. In the end, over 4600 students designed online implementations to test the model and reap good feedback.

The mobile Internet era facilitates daily life and generates a large amount of data and information at the same time, and most of the dazzling types of recommendation systems are used in the commercial field. Under the double pressure of work and study, the realization of the personalized recommendation of educational resources has become the primary problem that needs to be solved in intelligent education. The application of a personalized recommendation system can effectively solve the problem of cognitive overload or vagueness when users are learning online, which can greatly improve resource utilization and user learning efficiency. With the importance of personalized learning, personalized recommendations based on recommendation algorithms provide a good opportunity for the development of personalized learning. To improve online education, this paper investigates how to introduce the personalized recommendation technology widely used in the commercial field in online education and finally designs and implements a personalized education platform based on a collaborative filtering algorithm. On this basis, online teaching on this platform is divided into two modes: one is the original teacher uploads recorded teaching videos; students can learn by purchasing online or offline download; the other is interactive online live teaching; each course is a separate online classroom; the teacher will publish online class information in advance; students can purchase the classroom number and password information, online learning.

\section{Analysis of Online Education Course Recommendations by Collaborative Filtering Algorithm}

2.1. Improved Cofiltering Algorithm. Since the 1990s, after 30 years of changes, online education platforms with online education functions have emerged and increased people have been learning a variety of courses on the Internet. Since then, online learning has entered people's daily lives. In the original old system, the search function provided had to use specific and exact keywords to complete the search function. The advancement of technology has led to the development of stronger and more progressive online education features [19]. The number and variety of courses that can be viewed by users of learning today have shown massive growth. The rich and varied types of courses make it difficult to specifically categorize and find them. This phenomenon also prevents users from making accurate descriptions to search for keywords. As a result, it becomes difficult to search for the resources you need in the huge dataset. Given this situation and the growing needs of users, recommendation functions are gradually being integrated into the development of online education platforms. The integration of this recommendation feature enables users to select and recommend the course content they need among the online resources based on the characteristics of their needs [20]. When the demand is uncertain and the description of the project features is unclear, the recommendation system can also mine the user's records to analyze the most likely demand and provide the most appropriate demand recommendation to the user. With high-precision and high-efficiency recommendation functions, personalized recommendations greatly improve users' experience comfort and user stickiness and occupy an important position in daily life.

The content-based collaborative filtering recommendation algorithm uses a scoring matrix to calculate the similarity 
between users or items and makes recommendations based on the similarity relationship between items or users. Under the current research, content-based collaborative filtering recommendation algorithms can be composed of user-based collaborative filtering (UBCF) and item-based collaborative filtering (IBCF). Their hybrid technique is based on the basic idea of UBCF recommendation formation, recommending items with the same or similar interests and preferences. Collaborative filtering is a popular recommendation because it can deal with unstructured and complex objects. Current system prototypes include Rigor for music album recommendations, PHALS for Web message recommendations, Jester for joke recommendations, and Amazon for book recommendations [21]. Content-based recommendations firstly make similarities between computed users or items, to find similar users or items, i.e., neighboring users, and secondly score predictions by aggregating the scores of neighboring users. In the usual calculation method, the main intention of correcting cosine similarity as an upgrade of cosine similarity is to refine the cosine similarity and to consider only the problem of similarity in the direction of the vector dimensions, without considering the dimensional differences between the dimensions. Therefore, a correction operation of subtracting the mean from each dimension will be performed to calculate the similarity. Pearson's correlation coefficient is defined as the quotient and standard deviation of the covariance between two vectors.

$$
\begin{aligned}
& \operatorname{sim}(x, y)=\frac{\int_{i}^{y}\left(r_{x, y}-\overline{r_{x, y}}\right) \cdot\left(r_{y, x}-\overline{r_{y, x}}\right)}{\sum_{i e}\left(r_{x, y}-\overline{r_{x, y}}\right) \cdot\left(r_{y, x}-\overline{r_{y, x}}\right)^{2}} \\
& \operatorname{sim}(x, y)=\sin (\vec{x}, \vec{y})=\frac{\int_{i}^{y}\left(r_{x, y}-\overline{r_{x, y}}\right) \cdot\left(r_{y, x}-\overline{r_{y, x}}\right)}{\sqrt{\sum_{i e}\left(r_{x, y}-\overline{r_{x, y}}\right)^{2} \cdot\left(r_{y, x}-\overline{r_{y, x}}\right)}} .
\end{aligned}
$$

After completing the similarity calculation, the unknown evaluation is predicted by aggregating several neighbors, and the rating of item $s$ by user $x$ can be predicted by aggregating the evaluations of the most similar users of user $x$ [22].

$$
\overrightarrow{x_{c, s}}=\underset{c^{\prime} \in U}{\operatorname{aggr} x_{c, \sigma} .}
$$

The first aggregation function selects simple averages, the second aggregation function does not take into account the differences in user scoring styles (the scoring range is not limited by specific criteria), and the third aggregation function overcomes the above drawbacks by using the deviation of the scoring values from the corresponding user average scores when calculating weighted sums [23].

$$
\begin{aligned}
& \overrightarrow{x_{c, s}}=\frac{1}{\int_{i}^{y}(\operatorname{sim}(x, y)} \sum_{i e}^{\underset{c^{\prime} \in U}{\operatorname{agg} x_{c, s}},} \\
& \overrightarrow{x_{c, s}}=\frac{1}{\int_{i}^{y}(\operatorname{sim}(x, y)} \sum_{i e}^{\operatorname{aggrsim}\left(x, y^{\prime}\right) x_{c, s},} \\
& \overrightarrow{x_{c, s}}=\frac{1}{\int_{i}^{y}(\operatorname{sim}(x, y)} \sum_{i e} \operatorname{aggrsim}\left(x, y^{\prime}\right) \cdot\left(r_{y, x}-\overline{r_{y, x}}\right) x_{c, s} .
\end{aligned}
$$

To understand and figure out what the users need or want, the recommendation function mines and organizes the search records and further analyzes the user's behavior on the website to obtain some information about the user's preferences and then selects the data that meets the user's needs and the user's possible preferences from the many sources of data and recommends the data or products to the user [24]. The algorithm structure of the personalized recommendation system is shown in Figure 1.

First, the user accesses the front-end Web server, which contains the user's operations in the client, such as account registration, viewing, evaluation, purchase; the server sends this information to the matter processing server for processing; the matter processing server transcodes the information into structured information, then, through the data mining engine for further processing of the information, mining the information to establish a similar user model. On the one hand, the model is put into the model library to be retrieved by the personalized recommendation engine at any time, and, on the other hand, the information is processed and stored in the database for retrieval by the data processing server. Finally, the personalized recommendation engine will combine the data extracted from the data processing services with the model in the model library and perform algorithm calculation according to the algorithm formula and then recommend the items that users need to achieve the purpose of personalized recommendation. In summary, a perfect recommendation system mainly includes the following three parts: as the basis of the recommendation function, it is mainly used to organize and collect relevant information. Usually, the recommendation function collects relevant information from users in two ways: explicitly and implicitly. The dominant way is to give the user explicit feedback by purchasing a course, listening to music, watching a movie, or reading a book. As a dynamic behavior that changes with the user, it is the best way to get accurate information. The best way to get accurate information is to balance the explicit and implicit information acquisition, 


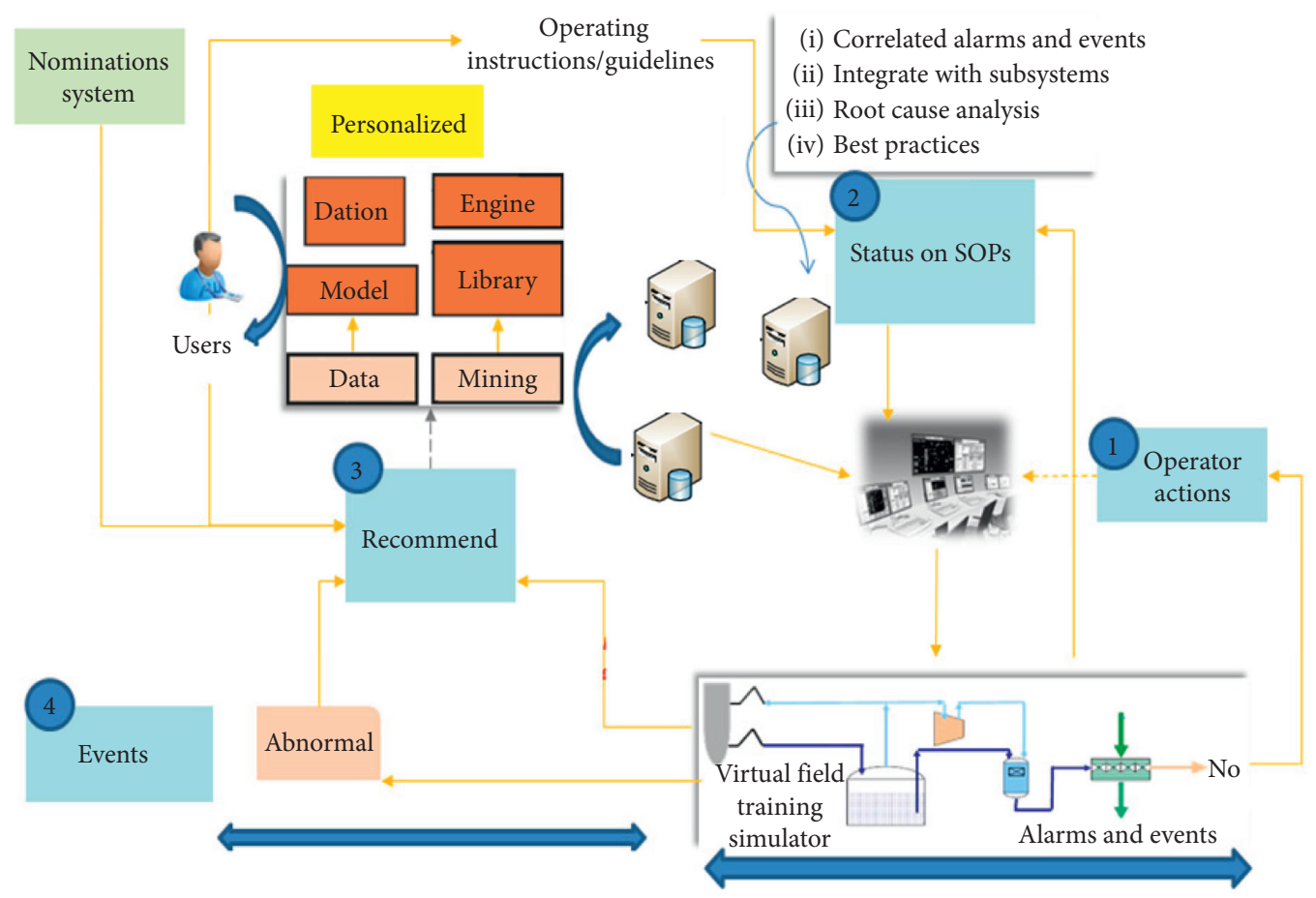

FIgURE 1: Personalized recommendation algorithm collection model.

which is the most suitable way to pursue high-efficiency recommendations by the high personalized recommendation system. The recommendation results are displayed in the recommendation interface, and the server returns the recommendation results to the user. As the main body of the recommendation system, one is to mine the user's records, that is, to mine the user's hidden data and information through the user's past behavior to realize the preprocessing of user information, which helps to reduce data sparsity and improve the real time and scalability of recommendations. It also generates and trains models to build multiple recommendation models that are valuable for improving the personalized recommendation system by mining the hidden information. Its design directly affects the final performance, and the soul of the recommendation function is accuracy and efficiency. The core part of the system is the personalized recommendation engine in Figure 1, which covers relevant personalized recommendation technologies and related algorithms. The comprehensive application of these related technologies and algorithms has a decisive influence on the accuracy and efficiency of the recommendation results, and the performance of the whole recommendation system can be improved by improving the relevant algorithms of this module.

2.2. Online Education System Design. Given the teaching functions of the platform, the client-side and server-side are designed separately, and the system is roughly divided into course management, user management, and comment management. As shown in Figure 2, the system is divided into three parts: course management, user management, and comment management.
This paper focuses on the personalized online learning platform based on collaborative filtering algorithms, with the main goal of breaking the limitations of time and space, increasing the ways and means of learning, and using novel user enthusiasm for learning and improving the efficiency of user learning [25]. Online learning is one of the more popular ways of learning that allows students who do not have the time or the conditions to attend face-to-face courses to understand and learn what they need to know through online courses. User-based collaborative filtering methods are used to uncover the past behavior of the target users. Items are browsed and collected to gain insight into what the user likes, mining for preference information, and then the system is searched for users with similar preferences to the user and a formula is used to calculate the similarity between users. Then, intercept the specified number of neighboring users by the similarity ranking and make predictions on the target user's rating of an item based on the neighboring user's rating value of an item. After the structure is listed, the intercepted items with higher predicted scores are recommended to users. Because there are no onerous constraints, many cases can be matched and many abstract items can be handled well to discover user preferences. A user-based collaborative filtering approach uses user rating information that is a specific explicit value. Identifying similar users to the target user is the most important part of the process and a prerequisite for further predictions and recommendations.

Hotspots are news or information that attracts the public's attention. On the web, if a news message goes unclicked for a long time, the message will not naturally be hot. Conversely, if the message has many clicks, it indicates that the message is hot. Hot recommendations reflect the 


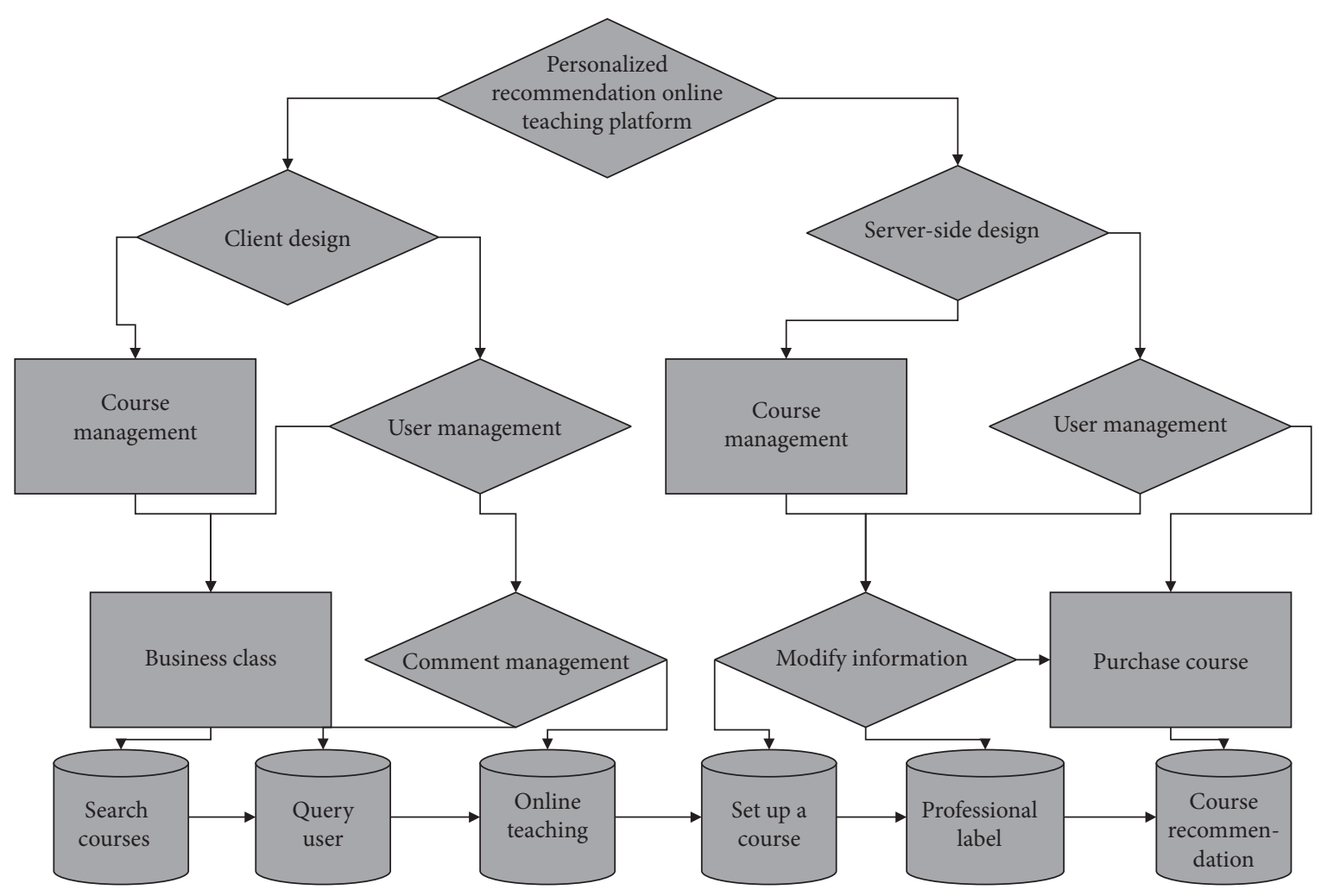

FIgURE 2: System function module diagram.

timeliness of the video site's content. Hot massage recommendations for video websites are a common method of recommending messages, providing users with the most popular videos. Popular videos are accepted as clickbait ensuring that the videos are popular and acceptable. Hot videos are usually set up as banners or pop-up windows on the home page, preset by the website staff, and manually updated to recommend combinations of popular videos. The successful application of hot recommendations on video websites inspired us to make recommendations for users based on the video website's hot recommendations model, which is based on the platform's hot courses. On the one hand, we recommend hot courses by counting the number of clicks and plays in a certain period and sorting them according to the number of clicks and then intercept the target number of hot courses on the user's home page. On the other hand, the platform will screen the courses with high evaluation based on the current industry trend and recommend the hot courses on the user's home page. As a kind of indiscriminate overlay recommendation method, hotspot recommendation has the features of simple operation, high public acceptance, and large click flow.

Students can click on the teacher's picture to go to the teacher's information page, which will show the teacher's style, teaching experience, and teaching direction. The course information will be entered into the database and displayed on the teacher login page and student login page; students can see that the course information will be intuitive to see the course name, the main teacher information, and the main content, both convenient and intuitive. All the information of the live online courses will be displayed in this module; in this module you can search the course information; we can view the online course description, course teacher, course time and duration, and the course fee; students can purchase online and join the course. There are two types of course recommendations: hot course recommendations and personalized course recommendations based on a collaborative filtering algorithm. In this module, hot courses will be recommended on the homepage by counting the number of times the course is played and selecting the current hot courses. It will also use the relevance algorithm to determine its neighboring users and recommend suitable courses for users based on the search content of each user.

The database is designed according to the teacher-student integrated online education process; the main categories of entities involved in the platform are user data (students and teachers), course data, exercise data, notes data, student question data, and learning resources data, where the exercise data relates to the student user answer behavior and learning resources data relates to the hotness of the learning resources available for popular recommendations, the user's learning resources.

The development environment used for the platform is MyEclipse, with the Tomcat server as the Web application server. All data is managed by MYSQL and integrated by the framework. After logging in, student users can learn through Web pages. The platform is realized from the back-end to the front-end, and after entering the platform there is a display of various functions of the platform. 
The digital library module is the recommended algorithm application part in the rehearsal platform; in the daily study, learning materials are indispensable. Digital library resources are mainly based on classification and retrieval, lacking personalized services and failing to focus on the collection and analysis of student user data to effectively understand the learning habits and content tendencies of student users. Based on the above problems, this module uses a mixed recommendation algorithm to make up for the lack of personalization and provide a personalized service of learning resources for thousands of student users. Books or handouts are recommended to student users based on their preferences and the learning preferences of students with similar levels of proficiency. New users will recommend the books and lectures with the highest popularity in the platform utilizing hot ranking, and those that meet the criteria of the recommendation algorithm will be recommended utilizing mixed recommendations for rationalization. The search section of the digital library module provides not only a keyword fuzzy search, but also an advanced search. In addition to the fuzzy keyword search, the search part of the digital library module also provides advanced search methods for users to search accurately. This part is the core part of the platform; first of all, the background is written; after creating the entity class, the layer starts to establish the method of adding, modifying, deleting, and viewing, then writes the service interface and implementation class, uses Spring annotation when developing, and then injects the mapper interface class into the service implementation class to complete the operation of adding, deleting, and deleting and changing the search. The service interface is injected into the controller layer and the methods in the interface are called, and the page information of the final query is passed to the interface of JSP through the view parsing layer. The final query page information data is passed to the interface of JSP through the view parsing layer. The add, modify, and delete operation is the right of the teacher user; the favorite and reading information of the student user has corresponding storage management in the database, and the description information is completed directly through the pop-up box; the download function of the student user is completed through the input stream and output stream.

\section{Analysis of Results}

3.1. Interactive Data Simulation Results. The data simulation process is shown in Figure 3. Firstly, user information is divided into different sets according to courses and traversed. In the original data, there are four-course results, where only Pass and Distinct are considered as Pass and Fail and Withdraw are considered as Fail. The set of passed students is then divided into four sets of 60-70, 70-80, $80-90$, and $90-100$ based on their scores. $10 \%, 15 \%, 20 \%$, and $25 \%$ of the users are randomly selected in each of the four sets, and they are assigned to be complimented. Among the complimented students, the number of compliments received is generated according to a normal distribution simulation, with four score band means defined as $\mu=10,15$,
20 , and 25 , respectively, with the same standard deviation taking $\sigma=3$.

The number of compliments is still normally distributed with mean $\mu=2$ and standard deviation $\sigma=2$. More than half of the students fail each course. When all compliments were assigned, all compliments in the same course were traversed, and, for each compliment, there was a $20 \%$ probability of generating interest. After traversing all the collections divided by course and then divided by region, users in the same region select $1 \%$ pairs for them to follow each other. The final data obtained is shown in Figure 3. Here, the data does not simulate the number of people who are clickstomped or reported, as this is rare in general online education platforms and there is no base strategy to generate these types of trust relationships.

The curve of accuracy and recall with similarity threshold $\sigma$ for user-based collaborative filtering recommendations using (2) is shown in Figure 4, where recall decreases with increasing $\sigma$ but is at a high-level overall, still 0.809 when $\sigma$ is taken 0.9. Accuracy increases overall with increasing $\sigma$ but is always at a low level, also only 0.809 when $\sigma$ is taken 0.9 . When $\sigma$ is very small, almost all other users' selected courses are recommended to the current user, which results in a recommended set of courses containing all courses in the dataset, leading to a high recall rate close to $100 \%$ and an accuracy close to the ratio of the average number of courses selected by the user to the total number of courses.

The f-score curve calculated using accuracy and recall is shown in Figure 4, which increases as $\sigma$ increases, and even $\sigma=1$ is the best recommendation, but this also means that the learner-based collaborative filtering recommendation is unsuccessful. The main reason for such a curve is that there are too few users attributes to differentiate users individually and there are only 7 courses in the dataset, which results in a high intersection between the number of courses recommended to users and the number of courses selected by users, even if the similarity threshold $\sigma$ is high. Also, there is a relationship between the values of certain characteristics, such as region and education, which cannot be adequately characterized by using unique hot codes. When the number of user vector dimensions increases, using memory-based collaborative filtering to calculate similarity may not be able to fully utilize the feature information.

Using a pairwise rate regression model for course selection prediction on the anonymized dataset, the final performance on the seven courses is shown in Figure 5, which reveals that the accuracy and recall rates are not very high. The observation of the training set shows that the cause of this phenomenon is the imbalance of positive and negative examples; linear and logistic regression and other models are very sensitive to the number of positive and negative examples of data when making bicategorical predictions, and the number of selections per course in the anonymized dataset is shown in Figure 5. The highest number of CCC course selections accounts for only $24.3 \%$ of the total records, while the lowest number of AAAs accounts for only $2.3 \%$, so you can see that the accuracy and recall of the AAA course selection predictions are significantly lower than the other course predictions. 


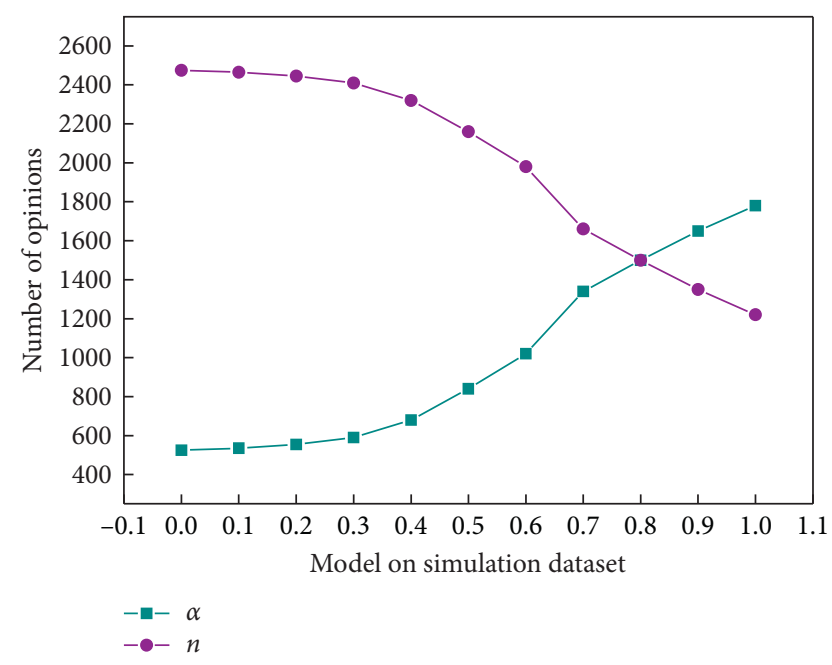

FIgURE 3: Plot of the total number of trustworthy compliments with distance decay parameter.

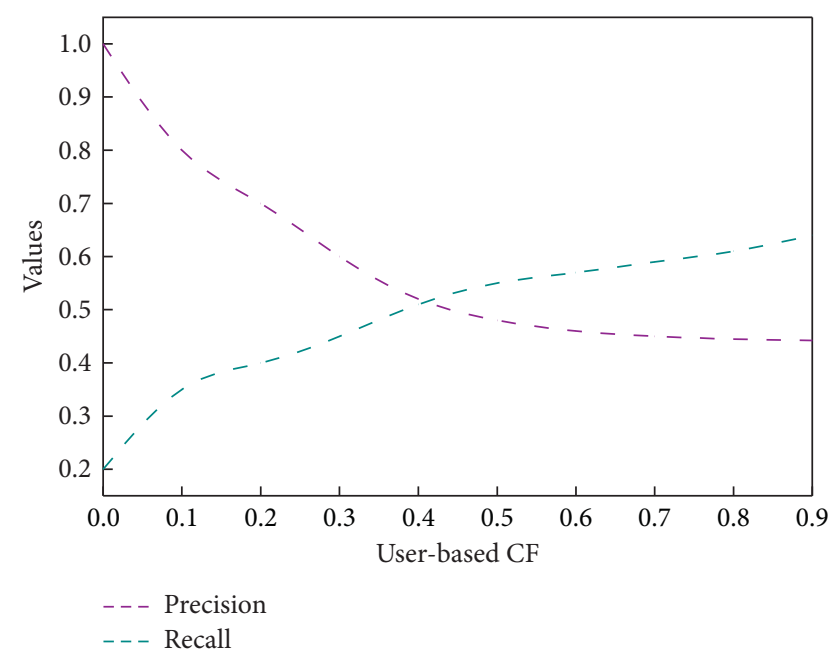

Figure 4: Plot of learner-based collaborative filtering recall vs. precision.

To solve this problem, before dividing the training set and test set, all positive examples of each course, i.e., all the records that selected the door, are taken out, and then an equal number of negative examples are randomly selected, and in this set with the equal number of positive and negative examples the training set and test set are divided using four triple-fold cross-validations for the training and validation of the LR categorization model. The final prediction results for the 7 courses are shown in Figure 6. The overall accuracy and recall rates are greatly improved, especially for course AAA, with the highest FYFFE reaching 81.3 accuracies and 79.2 recall rates.

Figure 6 shows the training of the LR model using only basic user attributes after removing the user habit label, and it can be seen that on all seven courses the prediction performance is worse than the model with personalized labels, which can prove that the inclusion of personalized labels can improve the accuracy of the

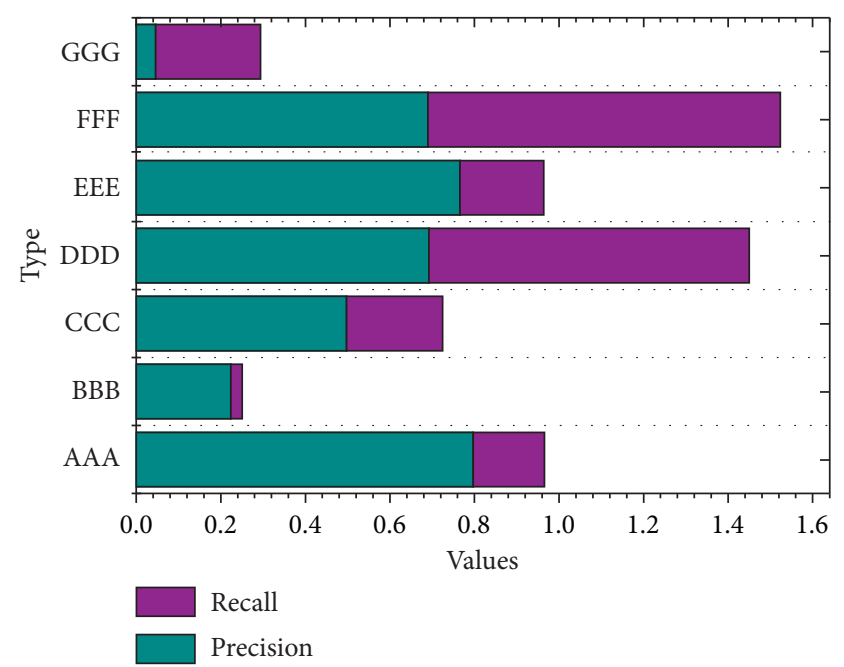

FIGURE 5: Average accuracy and recall for 7 courses from pair-rate regression.

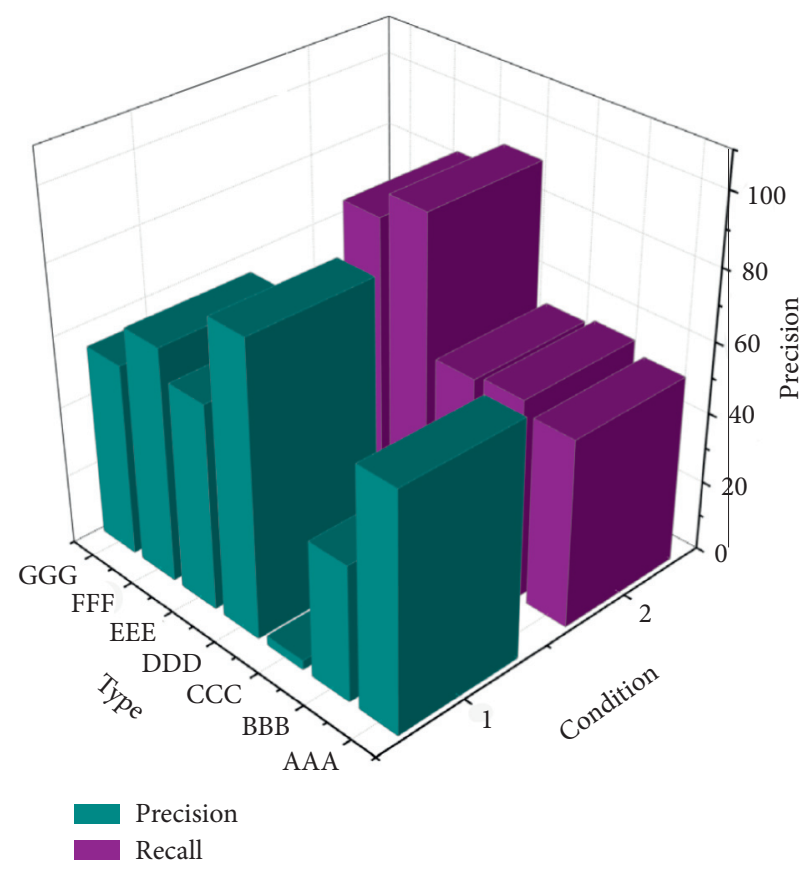

FIgURE 6: Average accuracy and recall rates using the balanced dataset on 7 courses.

recommendation model. Combining all the experiments in this section, it can be shown that the recommended model using log-rate regression is better than the learnerbased collaborative filtering on the anonymized data dataset, but the highest average f-score is only 80.24. There are several reasons for this: log-rate regression can only simulate linear combinatorial relationships between features, and more complex features can be more accurate. Combinatorial relationships may improve the effectiveness of the recommended model. The dataset is small, with an average of only 4656-course selection records per course, and even if experimental validation 
was performed using a subset of equally positive and negative examples, the accuracy of the model will still be affected by many counterexamples. The user portrait information is not rich enough or accurate enough, and only learning habit labels are currently used, and the learning habit labels do not take full advantage of all user behavior information and learning records in the dataset.

3.2. Experimental Results and Analysis. Experiments were conducted with $k=3, k=4$, and $k=5$, respectively. The accuracy line graph is shown in 7 , and the recall line graph is shown in 3-11; we can see that the accuracy and recall do not increase with the increase of the feature embedding vector dimension, but the difference between $k=4$ and $k=5$ in the two metrics is not large and even decreases in many courses. In this paper, the FM input feature vector dimension is only 28 , and the embedding vector does not need to be too complex. Although the regular term is added to prevent overfitting, the higher dimension still leads to a decrease in the prediction performance. Therefore, the embedding vector dimension $k$ of the feature is set to 4 in subsequent experimental comparisons and the development of FM-based recommended applications. Factor decomposer accuracy variation curve is shown in Figure 7.

The f-scores of the two models on the seven courses are shown in Figure 8. The prediction performance of FM is better than logistic regression on each course, proving that there is a correlation between the second-order combination of features and the prediction results. Also, it can be found that the prediction performance of AAA is poor on both models, which is mainly because there is less course-related data to distinguish between users who take and do not take courses. The best prediction, of course, FFF, is found in both models, although the number of courses taken is less than that of BBB, which may be due to the more obvious difference in the user characteristics of course FFF.

Problems in the experiments are analyzed and addressed, such as the influence of the special distribution of different data on the classification results and overfitting due to the high embedding dimension. The advantages and disadvantages of three recommended models, namely, memorybased collaborative filtering, log-likelihood regression, and factor decomposer, are evaluated on the dataset, and the factors that limit their performance are analyzed. The factor decomposer with the best performance is selected as the recommended model to support subsequent research.

The Teaching Overview module is designed to help teachers keep track of their students' learning, to check their students' learning levels, and to provide a hierarchical view of the class and student comparisons, as shown in Figure 9.

The online Q\&A and selection of good notes module are the teacher-student interaction part. For the students' questions, the teacher will answer the questions accordingly; for the students' open category notes, the best notes will be selected for more students to learn. Stress test is using JMeter, where the HTTP request is the server request

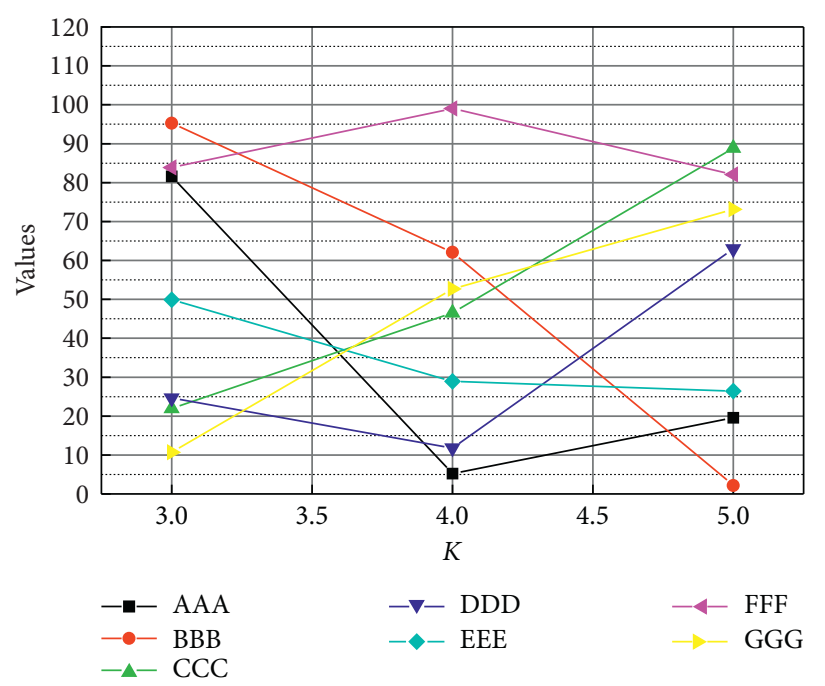

Figure 7: Factor decomposer accuracy variation curve.

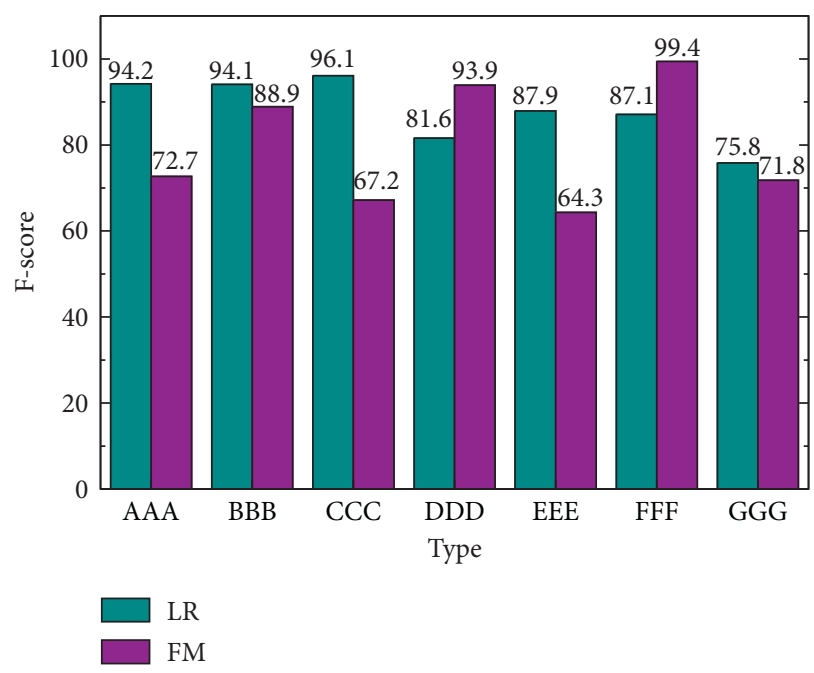

FIgURE 8: Comparison of the recommendation effect of pair-rate regression and factor decomposer.

timeout; while the access volume is about 100,000 orders of magnitude, the error rate is very low, which meets the daily access needs of the platform. The page response is checked, as shown in Figure 10.

An interface test using mail carrier is performed to check the first book using advanced search with keyword java, the server response is successful, and the response time is $322 \mathrm{~ms}$; the corresponding return value matches the predicted data. The outline design and database design of the rehearsal platform are introduced, and the basic functions of the rehearsal platform on the student side and the teacher side are demonstrated and explained, and the recommendation algorithm module on the student side is demonstrated and explained. Finally, tests are conducted on both the basic functions of the platform and the recommendation function module as well as the performance. The application of a suitable recommendation algorithm on the education platform can 


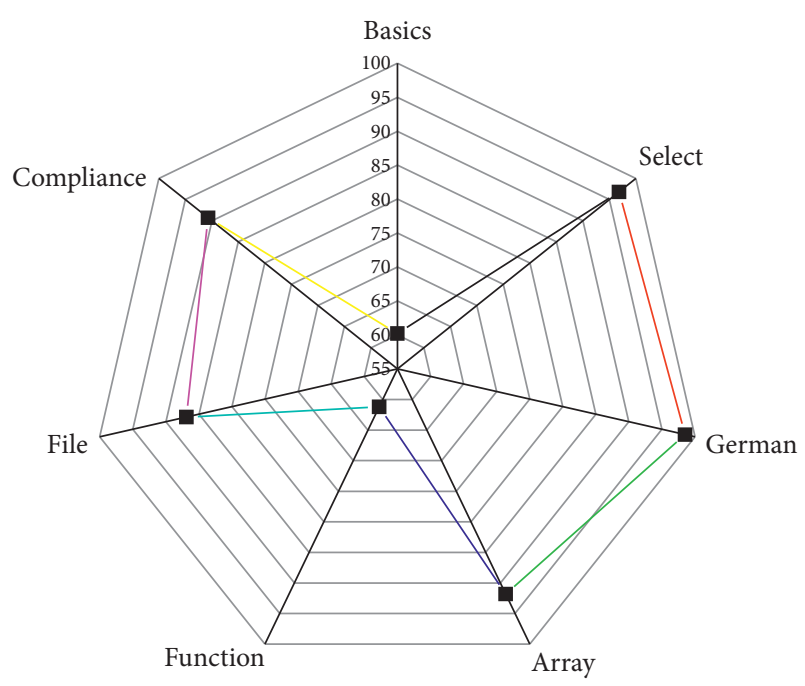

Figure 9: Level 3 Teaching Overview Page.

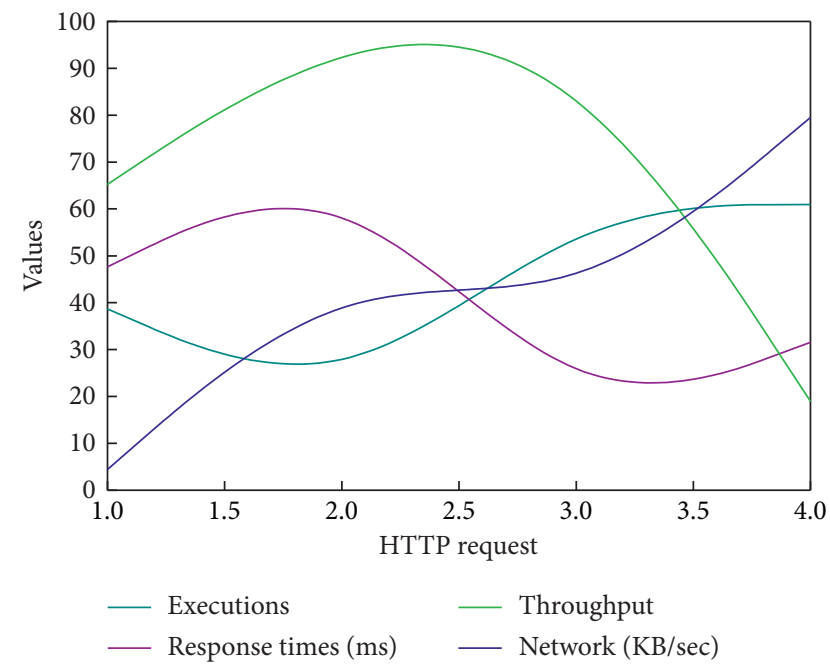

Figure 10: Stress test results.

accelerate the process of cultivating high-level professionals, and the addition of personalized recommendations on the education platform will create great business value.

\section{Conclusion}

Under the double pressure of work and study, the realization of the personalized recommendation of educational resources has become the primary problem that needs to be solved in intelligent education. As a key technology to solve the problem of cognitive overload or disorientation during online learning, the personalized recommendation system has become the focus of current research on how to combine with online teaching. In the platform design process, we have studied the application of various recommendation algorithms in practice and tried to achieve the actual effect of combining the recommendation algorithm with online teaching and finally selected a personalized course recommendation system based on the user's collaborative filtering algorithm. Initially, we achieved accurate and rapid course recommendations, which laid a foundation for personalized teaching. In this paper, when developing the rehearsal platform, we first do a multifaceted study on online education platforms and find that building personalized and efficient recommendations for educational resources has become a research hotspot of current education platforms and improve the shortcomings of traditional computer education platforms by increasing the interaction between school teachers and students and the detection of students' learning by teachers. Then, we studied and researched the contents related to the recommendation algorithm and designed a hybrid recommendation model. After a detailed system requirements analysis of the rehearsal platform, we completed the design and implementation of the rehearsal platform based on the hybrid recommendation algorithm by using the appropriate framework and development technology and the tendency of student users' learning habits using the recommendation algorithm. Finally, the rehearsal platform was tested for its functionality and performance, and the expected results were achieved. The rehearsal platform provides students with suitable IT learning resources and a platform for learning exercises, and the recommendation system not only helps users to quickly find high-quality information that interests them, but also saves their time and costs.

\section{Data Availability}

The data used to support the findings of this study are available from the corresponding author upon request.

\section{Conflicts of Interest}

The authors declare that they have no conflicts of interest reported in this paper.

\section{Acknowledgments}

This work was supported by the Teaching Reform and Research Projects in the 13th Five-Year Plan of Zhejiang Province (the Second Batch): Reconstruction of the Blended Teaching Mode of "One Center, Four Combinations and Three Reflections" under the Background of MOOC (no. jg20190301).

\section{References}

[1] J. Xiao, M. Wang, B. Jiang, and J. Li, "A personalized recommendation system with combinational algorithm for online learning," Journal of Ambient Intelligence and $\mathrm{Hu}$ manized Computing, vol. 9, no. 3, pp. 667-677, 2018.

[2] H. Zhang, T. Huang, Z. Lv, S. Liu, and Z. Zhou, "MCRS: a course recommendation system for MOOCs," Multimedia Tools and Applications, vol. 77, no. 6, pp. 7051-7069, 2018.

[3] Y. Pang, Y. Jin, Y. Zhang, and T. Zhu, "Collaborative filtering recommendation for MOOC application," Computer Applications in Engineering Education, vol. 25, no. 1, pp. 120-128, 2017. 
[4] J.-W. Han, J.-C. Jo, H.-S. Ji, and H.-S. Lim, "A collaborative recommender system for learning courses considering the relevance of a learner's learning skills," Cluster Computing, vol. 19, no. 4, pp. 2273-2284, 2016.

[5] C. Yin, L. Shi, R. Sun, and J. Wang, "Improved collaborative filtering recommendation algorithm based on differential privacy protection," The Journal of Supercomputing, vol. 76, no. 7, pp. 5161-5174, 2020.

[6] L. Xiaojun, "An improved clustering-based collaborative filtering recommendation algorithm," Cluster Computing, vol. 20, no. 2, pp. 1281-1288, 2017.

[7] V. Subramaniyaswamy, R. Logesh, M. Chandrashekhar et al., "A personalised movie recommendation system based on collaborative filtering," International Journal of High Performance Computing and Networking, vol. 10, no. 1-2, pp. 54-63, 2017.

[8] E. U. Okon, B. O. Eke, and P. O. Asagba, “An improved online book recommender system using collaborative filtering algorithm," International Journal of Computer Applications, vol. 179, no. 46, pp. 41-48, 2018.

[9] S. Yanhong, "Design of digital network shared learning platform based on SCORM standard," International Journal of Emerging Technologies in Learning (iJET), vol. 13, no. 07, pp. 214-227, 2018.

[10] A. Klasnja-Milicevic, M. Ivanovic, B. Vesin et al., "Enhancing e-learning systems with personalized recommendation based on collaborative tagging techniques," Applied Intelligence, vol. 48, no. 6, pp. 1519-1535, 2018.

[11] J. Chen, C. Zhao, L. Uliji, and L. Chen, "Collaborative filtering recommendation algorithm based on user correlation and evolutionary clustering," Complex and Intelligent Systems, vol. 6, no. 1, pp. 147-156, 2020.

[12] X. Liu, "A collaborative filtering recommendation algorithm based on the influence sets of e-learning group's behavior," Cluster Computing, vol. 22, no. 2, pp. 2823-2833, 2019.

[13] L. Jiang, Y. Cheng, L. Yang, J. Li, H. Yan, and X. Wang, “A trust-based collaborative filtering algorithm for E-commerce recommendation system," Journal of Ambient Intelligence and Humanized Computing, vol. 10, no. 8, pp. 3023-3034, 2019.

[14] M. K. Najafabadi, A. Mohamed, and C. W. Onn, "An impact of time and item influencer in collaborative filtering recommendations using graph-based model," Information Processing and Management, vol. 56, no. 3, pp. 526-540, 2019.

[15] O. C. Agbonifo and M. Akinsete, "Development of an ontology-based personalised E-learning recommender system," International Journal of Computer (IJC), vol. 38, no. 1, pp. 102-112, 2020.

[16] J. K. Tarus, Z. Niu, and D. Kalui, "A hybrid recommender system for e-learning based on context awareness and sequential pattern mining," Soft Computing, vol. 22, no. 8, pp. 2449-2461, 2018.

[17] P. Wei and L. Li, "Online education recommendation model based on user behavior data analysis," Journal of Intelligent and Fuzzy Systems, vol. 37, no. 4, pp. 4725-4733, 2019.

[18] Y. Tang and W. Wang, "A literature review of personalized learning algorithm," Open Journal of Social Sciences, vol. 06, no. 1, pp. 119-127, 2018.

[19] A. Kardan, A. Narimani, and F. Ataiefard, "A hybrid approach for thread recommendation in MOOC forums," International Journal of Social, Behavioral, Educational, Economic, Business and Industrial Engineering, vol. 11, no. 10, pp. 2195-2201, 2017.

[20] P. Dwivedi, V. Kant, and K. K. Bharadwaj, "Learning path recommendation based on modified variable length genetic algorithm," Education and Information Technologies, vol. 23, no. 2, pp. 819-836, 2018.

[21] M. Fu, H. Qu, Z. Yi et al., "A novel deep learning-based collaborative filtering model for recommendation system," IEEE Transactions on Cybernetics, vol. 49, no. 3, pp. 10841096, 2018.

[22] A. Al-Badarenah and J. Alsakran, "An automated recommender system for course selection," International Journal of Advanced Computer Science and Applications, vol. 7, no. 3, pp. 166-175, 2016.

[23] R. Logesh, V. Subramaniyaswamy, D. Malathi, N. Sivaramakrishnan, and V. Vijayakumar, "Enhancing recommendation stability of collaborative filtering recommender system through bio-inspired clustering ensemble method," Neural Computing and Applications, vol. 32, no. 7, pp. 2141-2164, 2020.

[24] Y. Yang, Y. Xu, E. Wang et al., "Improving existing collaborative filtering recommendations via serendipity-based algorithm," IEEE Transactions on Multimedia, vol. 20, no. 7, pp. 1888-1900, 2017.

[25] H. Li, H. Li, S. Zhang, Z. Zhong, and J. Cheng, "Intelligent learning system based on personalized recommendation technology," Neural Computing and Applications, vol. 31, no. 9, pp. 4455-4462, 2019. 